\title{
CNS Axons Globally Increase Axonal Transport after Peripheral Conditioning
}

\author{
Fernando M. Mar, ${ }^{1,2}$ Anabel R. Simões, ${ }^{1}$ Sérgio Leite,,${ }^{1,2}$ Marlene M. Morgado, ${ }^{1}$ Telma E. Santos, ${ }^{1}$ Inês S. Rodrigo, ${ }^{1}$ \\ Carla A. Teixeira, ${ }^{1}$ Thomas Misgeld, ${ }^{3}$ and Mónica M. Sousa ${ }^{1}$ \\ ${ }^{1}$ Nerve Regeneration Group, Instituto de Biologia Molecular e Celular (IBMC), University of Porto, 4150-180 Porto, Portugal, ${ }^{2}$ Instituto de Ciências \\ Biomédicas Abel Salazar (ICBAS), 4050-313 Porto, Portugal, and 3 Institute of Neuronal Cell Biology, Munich Cluster for Systems Neurology and German \\ Center for Neurodegenerative Diseases, Technische Universität München, 80802 München, Germany
}

Despite the inability of CNS axons to regenerate, an increased regenerative capacity can be elicited following conditioning lesion to the peripheral branch of dorsal root ganglia neurons (DRGs). By in vivo radiolabeling of rat DRGs, coupled to mass spectrometry and kinesin immunoprecipitation of spinal cord extracts, we determined that the anterograde transport of cytoskeleton components, metabolic enzymes and axonal regeneration enhancers, was increased in the central branch of DRGs following a peripheral conditioning lesion. Axonal transport of mitochondria was also increased in the central branch of Thy1-MitoCFP mice following a peripheral injury. This effect was generalized and included augmented transport of lysosomes and synaptophysin- and APP-carrying vesicles. Changes in axonal transport were only elicited by a peripheral lesion and not by spinal cord injury. In mice, elevated levels of motors and of polyglutamylated and tyrosinated tubulin were present following a peripheral lesion and can explain the increase in axonal transport induced by conditioning. In summary, our work shows that a peripheral injury induces a global increase in axonal transport that is not restricted to the peripheral branch, and that, by extending to the central branch, allows a rapid and sustained support of regenerating central axons.

Key words: axon regeneration; axonal transport; conditioning lesion

\section{Introduction}

Although in the adult CNS axon regeneration fails, some CNS axons can be prompted to regenerate. For example, the central branch of dorsal root ganglia neurons (DRGs) gains regenerative capacity when the peripheral branch is injured-a paradigm known as conditioning lesion (Neumann and Woolf, 1999). Numerous molecular pathways are regulated by conditioning lesion, but none alone is able to fully replicate its effect.

As neurons are highly polarized, many newly synthesized regeneration-associated genes (RAGs), and cytoskeleton proteins need to be transported anterogradely to the axon tip. In fact, the speed of axonal regeneration is similar to the rate of the slow component b (SCb) of anterograde transport, supporting the relevance of transport in sustaining regeneration (Wujek and

Received 0ct. 30, 2013; revised March 17, 2014; accepted March 21, 2014.

Author contributions: M.M.S. designed research; F.M.M., A.R.S., S.L., M.M.M., T.E.S., I.S.R., and C.A.T. performed research; T.M. contributed unpublished reagents/analytic tools; F.M.M., S.L., C.A.T., and M.M.S. analyzed data; F.M.M. and M.M.S. wrote the paper.

This work was funded by FEDER through COMPETE and by National funds through Fundação para a Ciência e a Tecnologia (FCT) under the Project FCOMP-01-0124-FEDER-017455 (HMSP ICT/0020/2010). F.M.M. and S.L. were supported by FCT (SFRH/BD/43484/2008 and SFRH/BD/72240/2010) and C.A.T. by Programa Ciência, funded by POPH-QREN and MCTES. We thank Dr. Vitor Costa (Instituto de Biologia Molecular e Celular, IBMC) for help with $2 D$ gels and Dr. Paula Sampaio (IBMC) for help with confocal microscopy. T.M. was supported by the Deutsche Forschungsgemeinschaft (Center for Integrated Protein Science Munich, EXC 114, and Munich Center for Systems Neurology, EX( 1010).

The authors declare no competing financial interests.

Correspondence should be addressed to Monica M. Sousa, Nerve Regeneration Group, IBMC, Rua do Campo Alegre 823, 4150-180 Porto, Portugal. E-mail: msousa@ibmc.up.pt.

DOI:10.1523/JNEUROSCI.4680-13.2014

Copyright $\odot 2014$ the authors $\quad 0270-6474 / 14 / 345965-06 \$ 15.00 / 0$
Lasek, 1983). Anterograde axonal transport is divided into the slow component a (SCa), which transports neurofilaments, tubulin, and microtubule-associated proteins, and SCb, which transports cytoplasmic proteins, such as glycolytic enzymes and actin, whereas vesicles and membranous organelles are transported in the fast component (Lasek et al., 1984). The motors of slow and fast components are similar, and the different rates result from intermittent pausing behavior of cargoes (Roy et al., 2007). Retrograde transport is also critical for regeneration, as it participates in injury signaling (Abe and Cavalli, 2008).

Despite the above evidence suggesting a central role of axonal transport during regeneration, the modulation of transport by injury is not well understood. Initial studies suggested that enhanced axonal growth after conditioning lesion was related to increased transport of cytoskeleton components (McQuarrie and Grafstein, 1982; Oblinger and Lasek, 1988; McQuarrie and Jacob, 1991). However, a more detailed interrogation using current approaches is missing. In this work we used the conditioning lesion to further understand whether axonal transport is regulated in conditions of increased axonal regeneration.

\section{Materials and Methods}

Surgeries. Wistar rats (8- to 10-week-old) were handled according to European Union rules. For sciatic nerve injury (SNI), the sciatic nerve was transected at the mid-thigh. For spinal cord injury (SCI), a dorsal hemisection was performed at T8. Rats with conditioning were subjected to SNI 1 week prior SCI.

Radiolabeling of DRG neurons. L4,5 DRGs of 8- to 10-week-old naive rats and of rats with SNI performed $1 \mathrm{~d}$ before ( $n=6$ rats/group), were 
injected with $\left[{ }^{35} \mathrm{~S}\right]$-methionine/cysteine $(2 \mu \mathrm{l}$ [22 $\left.\mu \mathrm{Ci}]\right)$ per DRG). Six days later, SCI was performed. One week later, spinal cords were collected from L5 up to the injury site, and segmented into 6 fragments. Each fragment was homogenized and counts per minute of $50 \mu \mathrm{g}$ of protein were measured. Two-dimensional gels of the spinal cord fragment L4,5 were performed and exposed to a phosphor screen for 5 weeks. Radioactive spots were trypsin digested and identified by MALDI-TOF/TOF.

Quantitative real-time PCR. L4,5 DRGs from 8- to 10-week-old naive rats and rats $1 \mathrm{~d}$ or 1 week following SNI were collected. Quantitative real-time PCR (qRT-PCR) was performed using iQ Supermix (Bio-Rad).

Immunoprecipitation. Wistar rats (8- to 10-week-old), with and without a conditioning SNI (performed 1 week before SCI), were subjected to SCI and 1 week later the spinal cord (T9-T12) was collected (pool of five animals per group). Samples were homogenized in PBS containing $0.3 \%$ Triton X-100, protease inhibitors (GE Healthcare), and $1 \mathrm{~mm}$ sodium orthovanadate. For immunoprecipitation, anti-kinesin heavy chain (Millipore) was used. As negative control, mouse IgG was used. The immune complex was collected using protein $G$ magnetic beads (GE Healthcare). For Western blots, antibodies against RhoGDI (1:2000; Santa Cruz Biotechnology), CRMP-5 (1:3000; Professor Améli-Moradi, Université Lyon1, France), pan 14-3-3 (1:1000; Cell Signaling Technology), and PGP9.5 (1:1000; AbD Serotec) were used.

Lentivirus production. HEK 293 T cells were transfected with packaging plasmids pPAX and pLP/VSVG (Dr. Relvas, IBMC) and a lentiviral vector (pLKO) coding for puromycin resistance and expressing the shRNA of interest (Sigma-Aldrich). Forty-eight hours later viral titration was done by infecting HEK $293 \mathrm{~T}$ cells.

Neurite outgrowth. DRGs from naive rats were isolated as described previously (Miranda et al., 2011). One day after plating, cells were infected with lentivirus (5000 IU per well) for $12-16 \mathrm{~h}$, and $24 \mathrm{~h}$ later treated with puromycin $(5 \mu \mathrm{g} / \mathrm{ml})$ for $48 \mathrm{~h}$. To confirm shRNA efficiency, qRT-PCR for RhoGDI, CRMP-5, and PGP9.5 was performed; a reduction of 85,60 , and $95 \%$ was obtained, respectively. For neurite outgrowth, cells were trypsinized, replated for $12 \mathrm{~h}$, and $\beta$ III-tubulin immunocytochemistry (1:2000; Promega) was done. At least 150 neurons per condition were traced using NeuronJ. For transfection, 200,000 cells were nucleofected (Amaxa 4D) with R18-EGFP or control plasmid (Dr. Fournier, McGill University, Canada). Cells recovered for $48 \mathrm{~h}$, and $12 \mathrm{~h}$ after being trypsinized and replated, neurite outgrowth was measured.

Mitochondrial transport. The MitoMouse line P (mice of either sex expressing a mitochondrial targeting sequence fused to CFP under the neuronal thy1 promoter; Misgeld et al., 2007) was used. DRG explants from 8- to 10-week-old mice subjected to SNI were analyzed $1 \mathrm{~d}$ or 1 week following injury. In additional experiments, MitoMice were subjected to SCI and 2 weeks later an SNI was performed; DRG explants were analyzed 1 week later. Naive animals served as controls. Explants of L4,5 DRGs with attached dorsal roots and peripheral branches were imaged for up to $2 \mathrm{~h}$. Transport of axonal mitochondria was analyzed by confocal microscopy with acquisition at $0.8 \mathrm{~Hz}$ for $5 \mathrm{~min}$. The number of transported mitochondria was determined as described previously (Misgeld et al., 2007). The percentage of moving mitochondria was evaluated in three different time frames per axon in at least 12 axons per condition. Mitochondria moving in 10 consecutive frames were used to calculate velocities in at least 50 mitochondria per condition. To image DRG explants from animals with SCI, injured axons were labeled with $1 \%$ cholera toxin B (CTB)-Alexa 488 (Invitrogen) by performing three injections (total volume $7 \mu \mathrm{l}$ ) in the injured dorsal caudal spinal cord, immediately following lesion. Four days later, CTB-Alexa 488-positive axons in the nerve branches of L4,5 DRGs were imaged.

Transport in DRG neurons. Neuron cultures from L4,5 DRGs of naive or conditioned mice (DRGs collected 1 week following SNI) of either sex were performed. Cells were either infected with baculovirus expressing synaptophysin-GFP (Invitrogen), incubated with $100 \mathrm{~nm}$ LysoTracker (Invitrogen), or nucleofected with APP-YFP (Dr. Kaether, Leibniz Institute for Age Research, Germany). Vesicle velocity was measured as above, and the number of moving APP-containing vesicles was determined as the number of vesicles crossing a vertical line in either direction per minute. The effect of rolipram (0.5 $\mu \mathrm{M}$ in DMSO) on axonal transport was tested in DRGs by analysis of synaptophysin and lysosome transport as described above.

Western blotting. One day or 1 week following SNI, mouse dorsal roots were collected. Naive animals served as controls. Five animals per group were used. Antibodies against kinesin heavy chain (1:500; Millipore), cytoplasmic dynein (1:250; Millipore), $\beta$-actin (1:5000; Sigma-Aldrich), $\alpha$-tubulin (1:1000; Sigma-Aldrich), acetylated tubulin (1:5000; SigmaAldrich), tyrosinated tubulin (1:2000; Arium), and polyglutamylated tubulin (1:4000; Adipogen) were used.

Statistical analysis. All values are mean \pm SEM. Student's $t$ test was used.

\section{Results}

A conditioning injury increases the expression and anterograde transport of newly synthesized axonal proteins

To test whether a conditioning injury increases the intrinsic ability of DRGs to regenerate by eliciting the synthesis and anterograde transport of RAGs, we revisited radiolabeling assays used to characterize the different components of anterograde transport (McQuarrie and Grafstein, 1982; Wujek and Lasek, 1983; Lasek et al., 1984; Oblinger and Lasek, 1988), but focusing on transport in the central branch, and coupling radiolabeling with mass spectrometry. In rat spinal cords where SNI preceded SCI (conditioning lesion group), increased amounts of radiolabeled proteins were consistently found (Fig. $1 A$ ), suggesting increased synthesis and/or anterograde transport. This increase was confirmed by $2 \mathrm{D}$ gel analysis of the L4,5 spinal cord fragment, where a generalized 2 - to 3 -fold increase in radiolabeled protein content was found in spinal cords of animals with conditioning lesion, when compared with animals where only SCI was performed (Fig. $1 B$ ). In naive spinal cords, the radiolabeled protein content was similar to that of spinal cords from animals with SCI, whereas that of conditioned animals was undistinguishable from animals with SNI only (data not shown). These data show that increased protein synthesis in DRGs and/or transport to the central branch is elicited by the priming peripheral lesion and that this increase is not recapitulated by a central lesion. The two most abundant proteins identified were cytoskeletal components, tubulin and actin (Fig. $1 B$, spots 2 and 5 , respectively). Most of the proteins corresponded to metabolic enzymes (spot $1, \mathrm{NADH}$ dehydrogenase, NDUS1; spot 2, catalase; spot 6, creatinine kinase; spot 8, glutamine synthase; spots 11 and 12, acetyl-CoA acetyltransferase, acyl coenzyme A thioester hydrolase, and prolyl isomerase; spots 14 and 15, malate dehydrogenase), among which several glycolytic enzymes were found (spot 10, fructose-bisphosphate aldolase C; spots 16 and 17, glyceraldehyde-3-phosphate dehydrogenase; spot 9, phosphoglycerate kinase 1; spot 7, $\alpha$-enolase; spots 3 and 4, pyruvate kinase isozymes; Fig. $1 B$ ). In addition, others included heat shock protein 70 (HSP-70; spot 1), CRMP-5 (spot 4), G-protein subunit 2 (GNB2; spot 13), annexins 2 and 5 (Anxa2 and Anxa 5; spots 16 and 18), sirtuin2 (Sirt2; spot 16), 14-3-3 proteins (isoforms $\zeta, \gamma, \theta, \zeta / \delta, \eta, \beta / \alpha$, and $\varepsilon$, spot 19), RhoGDI (spot 20), and the neuron-specific ubiquitin C-terminal hydrolase 1 (PGP9.5, spot 20; Fig. 1B). Of note, as our analysis was performed following radioactive labeling of DRGs, it was restricted to proteins contained in sensory axons in the spinal cord.

To determine whether the increase in radiolabeled proteins could arise from increased gene expression in DRGs, we evaluated the expression of selected proteins identified by 2D gel analysis, namely the cytoskeleton genes $\beta$ III-tubulin (tubulin) and $\beta$-actin (actin), the glycolytic enzyme GAPDH, and the genes coding for CRMP-5, 14-3-3 ( $\zeta$ isoform), RhoGDI, PGP9.5, HSP70, GNB2, Anxa2, Sirt2, Anxa5, and NDUS1. Most of the pro- 
A
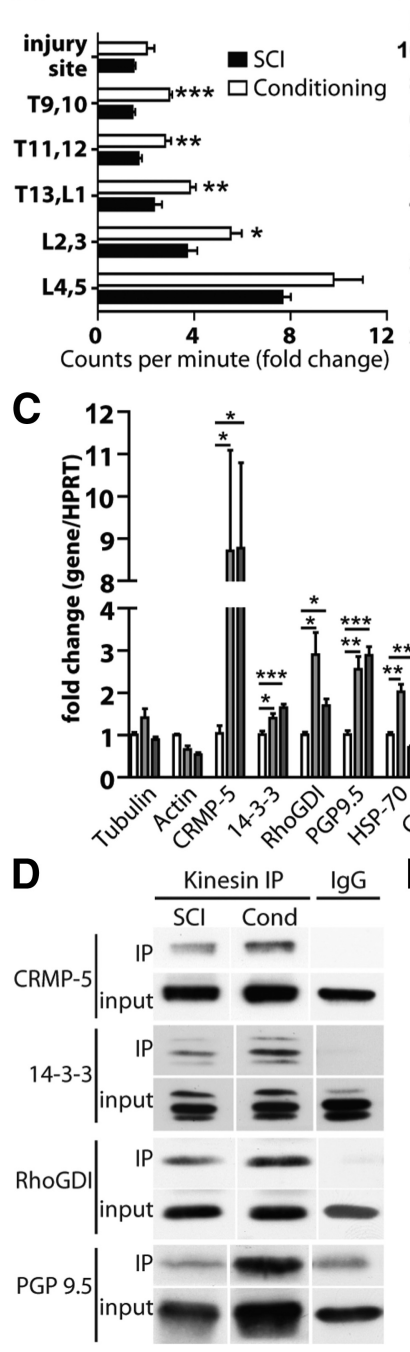
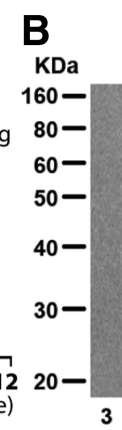

$\mathrm{SCl}$

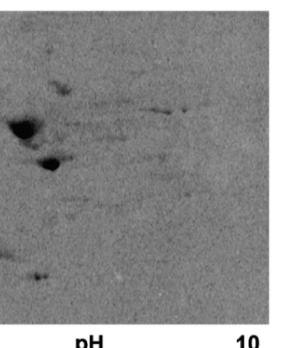

$\mathrm{pH}$

\section{$\square$ naïve $\square$ SNI 1d $\square$ SNI 1W}

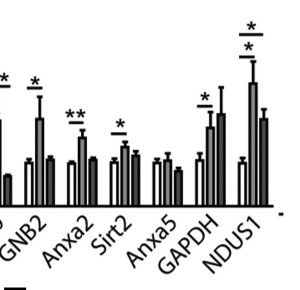

E

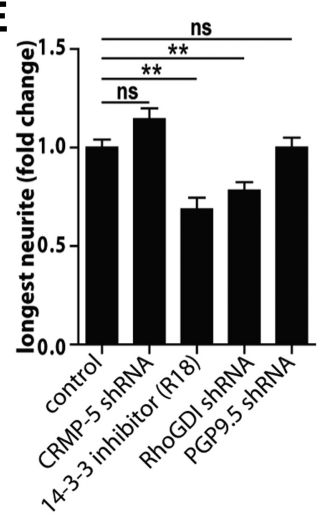

Figure 1. Conditioning lesion increases the synthesis and transport of proteins to the central branch of DRGs. $A$, Counts per minute in spinal cord fragments of rats with SCl or SCl preceded by SNI (conditioning). $B$, Representative $2 \mathrm{D}$ gels of the $L 4,5$ spinal cord fragment of rats with $\mathrm{SCl}$ or conditioning. C, qPCR of DRGs from naive rats, and from DRGs collected from rats $1 \mathrm{~d}$ or 1 week following SNI (SNI 1d and SNI 1w, respectively); $n=4$ naive, $n=5 \mathrm{SNI} 1 \mathrm{~d}$, and $n=10 \mathrm{SNI} 1 \mathrm{w}$. D, Representative Western blots of CRMP-5, 14-3-3, RhoGDI, and PGP9.5 following kinesin immunoprecipitation (IP) of spinal cords of animals with SCI or conditioning (Cond). $\boldsymbol{E}$, Neurite outgrowth of DRGs following transfection with R18, or infection with lentivirus expressing shRNA to CRMP-5, RhoGDI, or PGP9.5 $(n=3)$; ${ }^{*} p<0.05$, ${ }^{* *} p<0.01$, and ${ }^{* * *} p<0.001$.

teins had an increased expression $1 \mathrm{~d}$ after SNI, which in some cases was sustained at a later time point (1 week after SNI). Interestingly, however, others, including tubulin, actin, and Anxa5 had unchanged expression following injury (Fig. 1C). To test whether proteins identified by $2 \mathrm{D}$ gel analysis could also show increased anterograde transport in central axons from conditioned neurons, we performed kinesin-1 immunoprecipitation. In this experimental setup, all the axons of the spinal cord were analyzed, not just sensory axons. In spinal cords of conditioned animals, on average a twofold increase in the amounts of CRMP-5, 14-3-3, RhoGDI, and PGP9.5 that coimmunoprecipitated with kinesin-1 was found (Fig. 1D). This suggests that an increased association with the transport machinery occurs.

Although metabolic enzymes and cytoskeleton components are obvious candidates to be transported to the injury site, many of the remaining proteins identified have not been related to axonal regeneration. Thus, to determine their relevance, the ex- pression or activity of some of these proteins was inhibited in DRG neurons. We observed that neurite outgrowth was inhibited following shRNA-mediated knockdown of RhoGDI and by blocking the 14-3-3 proteins by transfection with R18, a pan 14-3-3 inhibitor (Kent et al., 2010), while the knockdown of PGP9.5 and CRMP-5 showed no significant effect (Fig. 1E). This suggests that at least for some of the proteins identified by 2D gel analysis, including RhoGDI and 14-3-3, synthesis in the DRG and anterograde transport through the spinal cord may contribute to the increased regenerative capacity induced by conditioning injury.

\section{Transport of mitochondria in the central branch of DRGs is increased following peripheral injury}

To determine whether axonal transport of organelles was also affected by a conditioning lesion, we used explants of DRGs from MitoMice (Misgeld et al., 2007) and imaged mitochondrial movements in the attached nerves. A peripheral injury increased the number of transported mitochondria, not only in the peripheral branch, but also in the central branch (Fig. $2 A-C)$. This increase was found both in the anterograde (Fig. $2 B$ ) and retrograde (Fig. 2C) direction. A 1.5-fold increase in anterograde transport was already observed at $1 \mathrm{~d}$ following SNI and was sustained 1 week following injury (Fig. 2B). In the case of retrograde transport, an increase was only detected 1 week following injury (Fig. 2C). At this time point, a conditioning injury also increased the percentage of moving mitochondria both in the peripheral and in the central branch (Fig. 2D). Although the conditioning injury led to an increased number of transported mitochondria in the central branch, no increase in the speed of transport was observed (Fig. 2 E,F).

To evaluate if an injury to the central branch triggered a similar effect in axonal transport, we injected fluorophoreconjugated CTB in the spinal cord immediately following SCI in MitoMice, which allowed the identification of axons affected by SCI (CTB-Alexa 488-positive; Fig. 2G). Injury to central axons failed to produce the same effect as the conditioning injury, since increased transport in the peripheral branch was not observed (Fig. $2 A, B$ ), and a 2.3 -fold decrease in the percentage of motile mitochondria (Fig. $2 D$ ), relative to naive animals, was found. In the central branch, SCI did not produce changes in mitochondria transport (Fig. 2B-D).

Peripheral injury performed after a central injury still increases the regenerative capacity of DRGs (Ylera et al., 2009). In MitoMice where SNI was done 2 weeks after SCI, transport was increased similarly to animals with SNI alone. An increased number of moving mitochondria (Fig. $2 A-C$ ) and an increased percentage of motile mitochondria (Fig. $2 D$ ) were observed. Overall, 


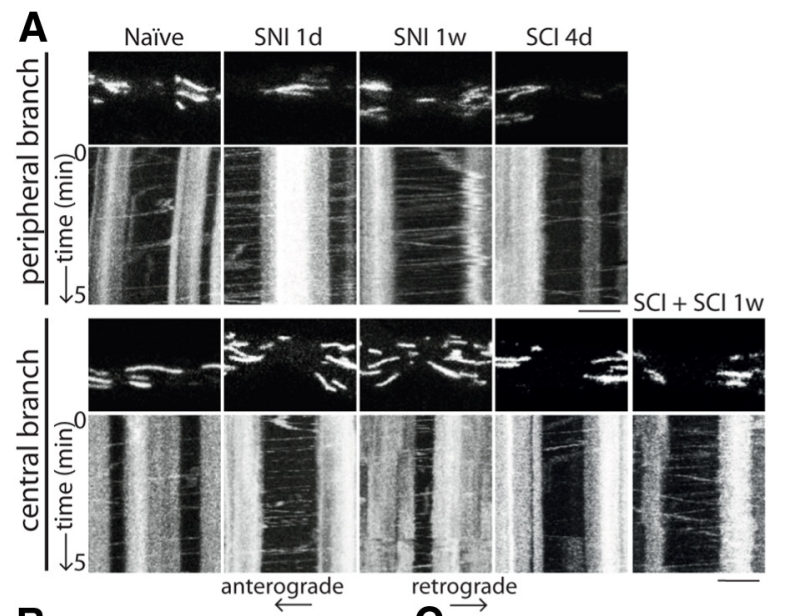

B

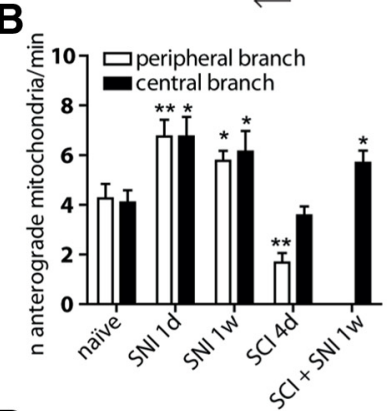

\section{D}
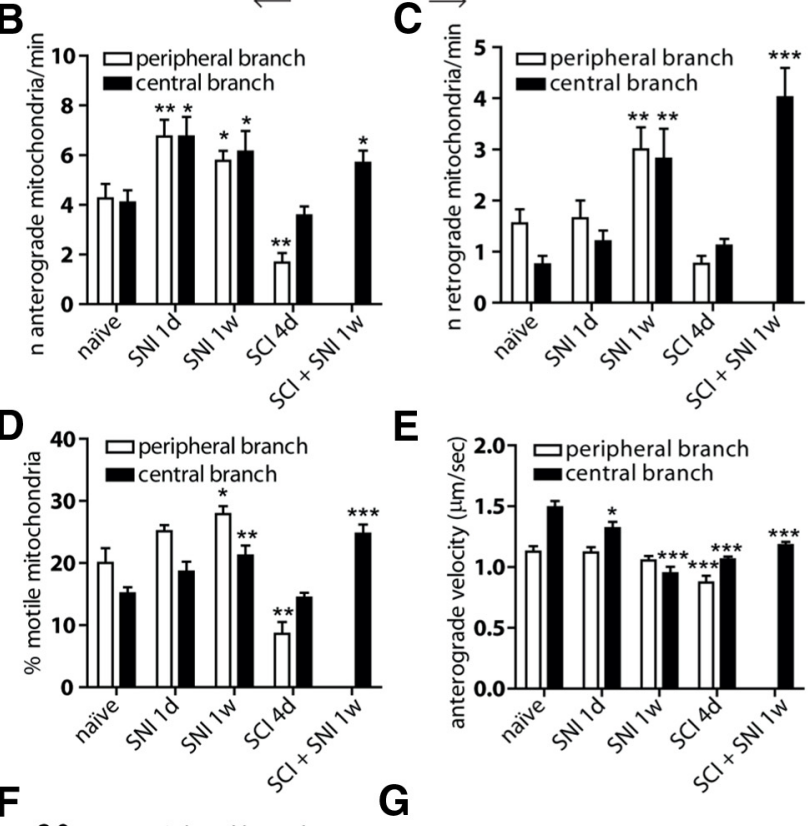

$\mathbf{E}$

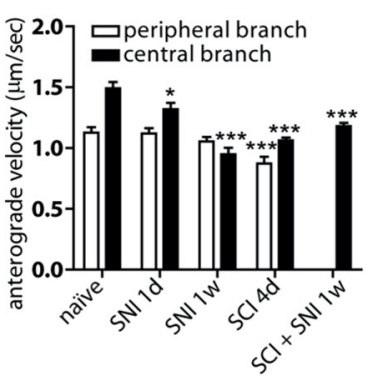

G

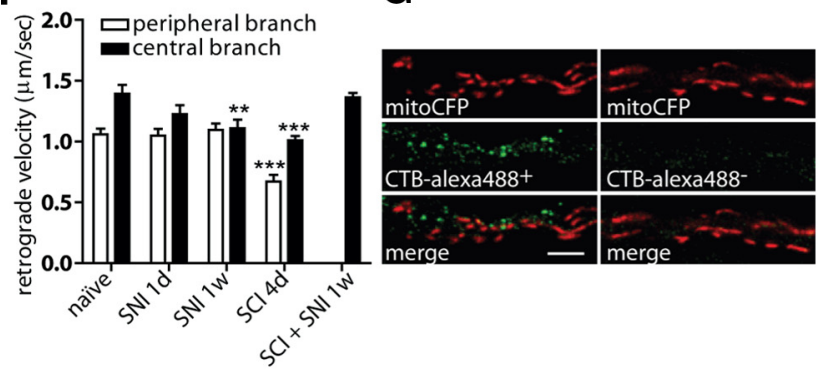

Figure 2. A conditioning injury increases the axonal transport of mitochondria. $\boldsymbol{A}, \mathrm{Ky}$ mographs of DRG explants from naive MitoMice, MitoMice where SNI was performed $1 \mathrm{~d}$ or 1 week before (SNI 1d and SNI 1w, respectively), MitoMice where SCI was performed $4 \mathrm{~d}$ before (SCI $4 \mathrm{~d}$ ), or MitoMice where SNI was performed 2 weeks after $\mathrm{SCl}$ and analysis was done 1 week later (SCI + SNI 1w; only the central branch was imaged). Top, Peripheral branch. Bottom, Central branch. Large vertical bands correspond to stationary mitochondria and fine diagonal lines are single motile mitochondria. $\boldsymbol{B}-\boldsymbol{F}$, Number of $(\boldsymbol{B})$ anterogradely and $(\boldsymbol{C})$ retrogradely moving mitochondria, $(\boldsymbol{D})$ percentage of moving mitochondria, and speed of $(\boldsymbol{E})$ anterogradely and $(\boldsymbol{F})$ retrogradely moving mitochondria in the peripheral and central branches of DRG explants from naive and injured MitoMice (SNI 1d, SNI 1w, SCI 4d, and SCI + SNI 1w). G, Representative micrographs of peripheral DRG axons of MitoMice injected with CTB-Alexa 488 in the $\mathrm{SCl}$ site, $4 \mathrm{~d}$ before. CTB-Alexa 488-positive (left) and -negative (right) axons are shown. Scale bar, $5 \mu \mathrm{m}$. only a peripheral injury (even if performed after central injury) elicits increased transport of axonal mitochondria.

In DRGs, a conditioning injury leads to increased transport of lysosomes and of synaptophysin- and APP-containing vesicles

To evaluate how broad the alterations in axonal transport induced by conditioning lesion would be, we assessed axonal transport of lysosomes, synaptophysin, and APP in DRGs from either naive or conditioned animals. In DRGs labeled with LysoTracker, the speed of transport was 1.6-fold increased specifically in the anterograde direction (Fig. $3 A, B$ ), whereas a conditioning injury did not alter the percentage of moving lysosomes (naive: $40.1 \pm$ 3.0\%; conditioning: $40.3 \pm 3.4 \%$ ). In DRGs transduced with synaptophysin, similar changes were observed. Whereas no differences were detected in the percentage of moving synaptophysin-containing vesicles (naive: $63.9 \pm 3.5 \%$; conditioning: $56.0 \pm 4.0 \%$ ), a 1.5 -fold increase in the speed of anterograde transport of synaptophysin was found in conditioned DRGs (Fig. 3C,D). Following APP-YFP transfection, a 2.2-fold increase in the number of anterogradely transported APPcontaining vesicles (Fig. $3 E, G$ ), and a 1.3-fold increase in speed of transport in both directions, were detected (Fig. $3 F$ ). In summary, a conditioning injury leads to broad alterations in axonal transport, affecting the transport of proteins, organelles, and vesicles, particularly in the anterograde direction.

Increased levels of molecular motors and of polyglutamylated tubulin can underlie the early increase in axonal transport elicited by a conditioning lesion

To understand the mechanism underlying the increased axonal transport in the central branch of conditioned DRGs, we analyzed whether conditioning would elicit alterations previously associated to the control of axonal transport, namely in molecular motor availability and suitable microtubular tracks. In DRGs, SNI was accompanied by 1.5- and 1.8-fold increased RNA levels of kinesin-1B and dynein-1, respectively, as early as $1 \mathrm{~d}$ following SNI (Fig. 4A). In the case of kinesin-1B, this increase was not sustained, but dynein-1 was still increased at 1 week following SNI (Fig. 4A). At the protein level, only kinesin-1 was significantly increased in the dorsal root $1 \mathrm{~d}$ following SNI but not 1 week following SNI (Fig. 4B). The anterograde transport of cargoes can be regulated by phosphorylation of kinesin light chain 1 (KLC1), as increased phosphorylation leads to dissociation of cargoes from the transport complex (Morfini et al., 2004). However, decreased levels of dephosphorylated KLC1 were present in dorsal roots 1 week after SNI (Fig. 4C), failing to explain increased anterograde transport.

Since tubulin modifications can alter the binding affinity of motors to microtubules, we tested whether changes in tubulin in the dorsal root could explain the conditioning-induced transport alterations. Tyrosination of tubulin is a marker of dynamic microtubules and can inhibit kinesin-1 binding to microtubules (Dunn et al., 2008). Interestingly, increased levels of tyrosinated tubulin were found $1 \mathrm{~d}$ and 1 week following conditioning injury (Fig. 4D,E), suggesting a more dynamic state of dorsal root microtubules. Tubulin acetylation has been associated with increased anterograde transport given improved kinesin-1 binding to microtubules (Reed et al., 2006). However, no alterations in tubulin acetylation were found (Fig. 4D,F). As kinesin-1 also binds more effectively to polyglutamylated tubulin (Ikegami et al., 2007), this modification was subsequently evaluated. Indeed, $1 \mathrm{~d}$ following conditioning injury, polyglutamylated tubulin was 
A
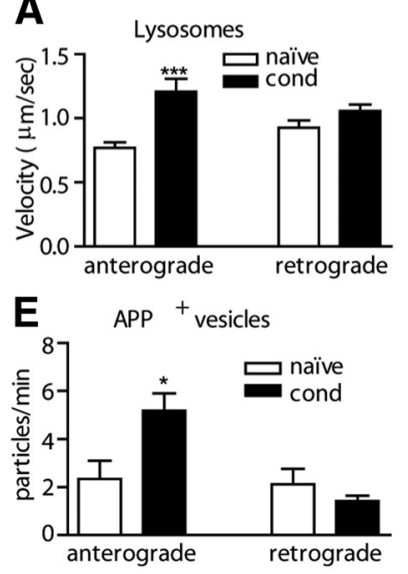
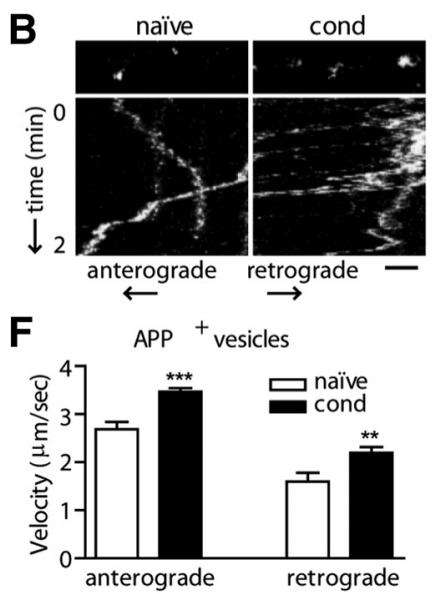
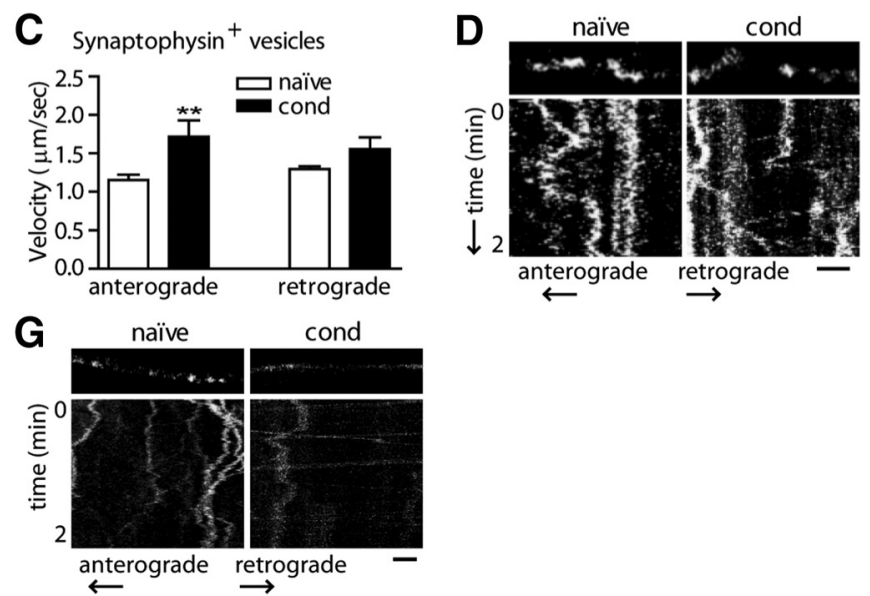

Figure 3. Conditioning injury increases the transport of lysosomes and of synaptophysin- and APP-containing vesicles. $\boldsymbol{A}$, Velocity of anterogradely and retrogradely moving lysosomes in naive and conditioned DRGs (at least 48 lysosomes per condition). $\boldsymbol{B}$, Kymographs of DRGs from naive or conditioned (cond) animals with lysosomes labeled with LysoTracker. Scale bar, $2 \mu$ m. $\boldsymbol{C}$, Velocity of anterogradely and retrogradely moving synaptophysin-positive vesicles in naive and conditioned DRGs (at least 44 synaptophysin-positive vesicles per condition). $\boldsymbol{D}$, Kymographs of DRGs from naive or conditioned animals transduced with synaptophysin-GFP. Scale bar, $2 \mu \mathrm{m}$. $\boldsymbol{E}$, Number of anterogradely and retrogradely moving APP-positive vesicles in naive and conditioned DRGs. $\boldsymbol{F}$, Velocity of anterogradely and retrogradely moving APP-positive vesicles in naive and conditioned DRGs (at least 42 APP-positive vesicles per condition). G, Kymographs of DRGs from naive or conditioned animals transfected with APP-YFP. Scale bar, $2 \mu \mathrm{m}$. ${ }^{*} p<0.05,{ }^{* *} p<0.01$, and ${ }^{* * *} p<0.001$.
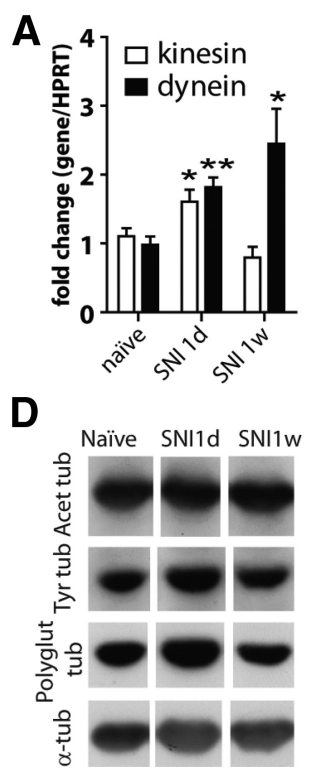

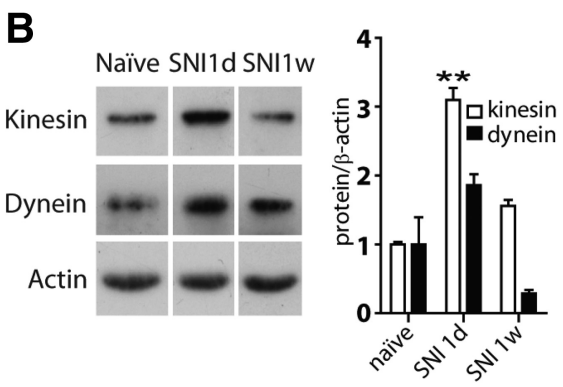

E

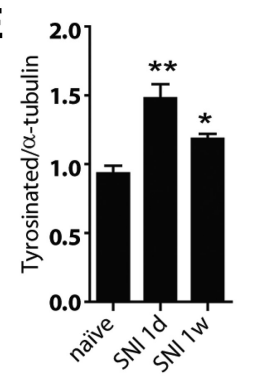

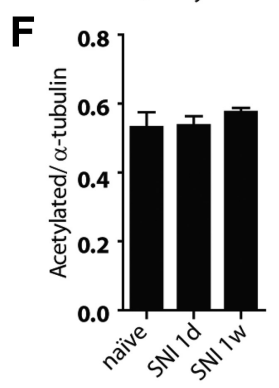

Figure 4. Increased levels of molecular motors and of polyglutamylated and tyrosinated tubulin are elicited following conditioning lesion. $\boldsymbol{A}, \mathrm{qPCR}$ of kinesin and dynein expression in DRG from either naive rats or collected from rats $1 \mathrm{~d}$ or 1 week following SNI (SNI 1d and SNI 1w, respectively; $n=7$ naive, $n=4$ SNI 1d, and $n=7$ SNI 1w). $\boldsymbol{B}$, Representative Western blots and quantification of kinesin and dynein in $L 4,5$ dorsal roots from either naive rats or collected from SNI $1 \mathrm{~d}$ and SNI $1 \mathrm{w} ; n=4 / \mathrm{group}$. C, Quantification of Western blots of dephosphorylated KLC in L4,5 dorsal roots from either naive rats or collected from SNI 1d and SNI 1w; $n=5$ /group. $D$, Representative Western blot analysis of tubulin in L4,5 dorsal roots from either naive rats or collected from SNI $1 \mathrm{~d}$ and SNI $1 \mathrm{w} ; n=5$ /group. $\boldsymbol{E}-\mathbf{G}$, Quantification of $\boldsymbol{D} ;{ }^{*} p<0.05$ and ${ }^{* *} p<0.01$.

increased in dorsal roots (Fig. 4D, G). However, this alteration was not sustained (Fig. 4G). In summary, increased levels of kinesin and polyglutamylated tubulin can underlie, at least partially, the early increase in anterograde axonal transport elicited by conditioning lesion.

\section{Discussion}

We show that in addition to broad changes in expression, a peripheral injury induces a global increase in axonal transport. Consequently, once a central injury occurs, any regenerative effort of the central branch can be rapidly supported. While in-

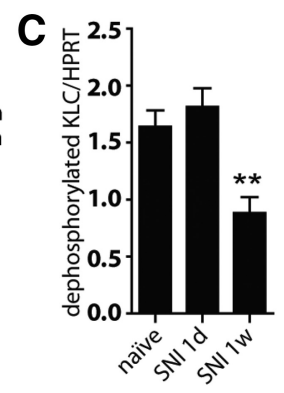

G

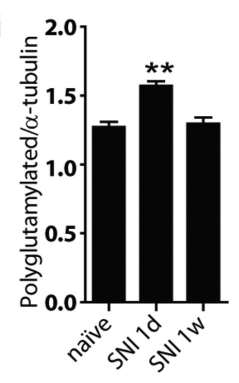

creased cAMP is a central mediator of the conditioning effect (Qiu et al., 2002), cAMP analogs fail to reproduce full conditioning (Blesch et al., 2012). Interestingly, cAMP is not able to increase axonal transport (Han et al., 2004). Rolipram, a phosphodiesterase inhibitor used to increase cAMP and promote CNS regeneration (Nikulina et al., 2004), did not alter transport in DRGs (data not shown).

We identified several glycolytic enzymes with increased anterograde transport in central axons following conditioning lesion. Interestingly, glycolysis is a central source of energy for vesicle transport (Zala et al., 2013). Transport of cytoplasmic proteins in $\mathrm{SCb}$ occurs through intermittent association to membrane-bound vesicles (Tang et al., 2012). Thus, the higher abundance of glycolytic enzymes, together with the increased transport of vesicles following conditioning lesion, may explain the increased transport of axonal proteins identified here.

Our approach allowed the identification of transported putative regeneration enhancers, such as 14-3-3 and RhoGDI. Supporting its relevance during axonal growth, 14-3-3 regulates axon guidance during development (Kent et al., 2010). Similarly, RhoGDI may be critical to regenerating axons as it inhibits RhoA, a central mediator of growth cone collapse (Sasaki and Takai, 1998). Surely, other proteins identified remain as interesting candidates to evaluate during axonal regeneration.

Molecular motors and polyglutamylated tubulin were increased in the dorsal root shortly after a peripheral lesion. As these effects were transient, the sustained increase of axonal transport at later time points remains to be explained. After conditioning lesion, the levels of tyrosinated, likely dynamic microtubules, were persistently increased. A precise control of 
microtubule dynamics is needed to optimize axon regeneration. Indeed, PNS neurons require decreased microtubule stability in the growth cone to regenerate (Cho and Cavalli, 2012). Hence, persistent alterations in microtubule stability might explain some of the effects observed.

Our work stressed that understanding the intersection between injury-induced signaling and axonal transport is an important aspect to consider. In summary, we propose that a broad increase in axonal transport is a major player during axon regeneration.

\section{References}

Abe N, Cavalli V (2008) Nerve injury signaling. Curr Opin Neurobiol 18: 276-283. CrossRef Medline

Blesch A, Lu P, Tsukada S, Alto LT, Roet K, Coppola G, Geschwind D, Tuszynski MH (2012) Conditioning lesions before or after spinal cord injury recruit broad genetic mechanisms that sustain axonal regeneration: superiority to camp-mediated effects. Exp Neurol 235:162-173. CrossRef Medline

Cho Y, Cavalli V (2012) HDAC5 is a novel injury-regulated tubulin deacetylase controlling axon regeneration. EMBO J 31:3063-3078. CrossRef Medline

Dunn S, Morrison EE, Liverpool TB, Molina-París C, Cross RA, Alonso MC, Peckham M (2008) Differential trafficking of Kif5c on tyrosinated and detyrosinated microtubules in live cells. J Cell Sci 121:1085-1095. CrossRef Medline

Han PJ, Shukla S, Subramanian PS, Hoffman PN (2004) Cyclic AMP elevates tubulin expression without increasing intrinsic axon growth capacity. Exp Neurol 189:293-302. CrossRef Medline

Ikegami K, Heier RL, Taruishi M, Takagi H, Mukai M, Shimma S, Taira S, Hatanaka K, Morone N, Yao I, Campbell PK, Yuasa S, Janke C, Macgregor GR, Setou M (2007) Loss of alpha-tubulin polyglutamylation in ROSA22 mice is associated with abnormal targeting of KIF1A and modulated synaptic function. Proc Natl Acad Sci U S A 104:3213-3218. CrossRef Medline

Kent CB, Shimada T, Ferraro GB, Ritter B, Yam PT, McPherson PS, Charron F, Kennedy TE, Fournier AE (2010) 14-3-3 proteins regulate protein kinase a activity to modulate growth cone turning responses. J Neurosci 30:14059-14067. CrossRef Medline

Lasek RJ, Garner JA, Brady ST (1984) Axonal transport of the cytoplasmic matrix. J Cell Biol 99:212s-221s. CrossRef Medline

McQuarrie IG, Grafstein B (1982) Protein synthesis and axonal transport in goldfish retinal ganglion cells during regeneration accelerated by a conditioning lesion. Brain Res 251:25-37. CrossRef Medline

McQuarrie IG, Jacob JM (1991) Conditioning nerve crush accelerates cytoskeletal protein transport in sprouts that form after a subsequent crush. J Comp Neurol 305:139-147. CrossRef Medline
Miranda CO, Teixeira CA, Liz MA, Sousa VF, Franquinho F, Forte G, Di Nardo P, Pinto-Do ÓP, Sousa MM (2011) Systemic delivery of bone marrow-derived mesenchymal stromal cells diminishes neuropathology in a mouse model of Krabbe's disease. Stem cells 29:1738-1751. CrossRef Medline

Misgeld T, Kerschensteiner M, Bareyre FM, Burgess RW, Lichtman JW (2007) Imaging axonal transport of mitochondria in vivo. Nat Methods 4:559-561. CrossRef Medline

Morfini G, Szebenyi G, Brown H, Pant HC, Pigino G, DeBoer S, Beffert U, Brady ST (2004) A novel CDK5-dependent pathway for regulating GSK3 activity and kinesin-driven motility in neurons. EMBO J 23:22352245. CrossRef Medline

Neumann S, Woolf CJ (1999) Regeneration of dorsal column fibers into and beyond the lesion site following adult spinal cord injury. Neuron 23:8391. CrossRef Medline

Nikulina E, Tidwell JL, Dai HN, Bregman BS, Filbin MT (2004) The phosphodiesterase inhibitor rolipram delivered after a spinal cord lesion promotes axonal regeneration and functional recovery. Proc Natl Acad Sci U S A 101:8786-8790. CrossRef Medline

Oblinger MM, Lasek RJ (1988) Axotomy-induced alterations in the synthesis and transport of neurofilaments and microtubules in dorsal root ganglion cells. J Neurosci 8:1747-1758. Medline

Qiu J, Cai D, Dai H, McAtee M, Hoffman PN, Bregman BS, Filbin MT (2002) Spinal axon regeneration induced by elevation of cyclic AMP. Neuron 34:895-903. CrossRef Medline

Reed NA, Cai D, Blasius TL, Jih GT, Meyhofer E, Gaertig J, Verhey KJ (2006) Microtubule acetylation promotes kinesin-1 binding and transport. Curr Biol 16:2166-2172. CrossRef Medline

Roy S, Winton MJ, Black MM, Trojanowski JQ, Lee VM (2007) Rapid and intermittent cotransport of slow component-b proteins. J Neurosci 27: 3131-3138. CrossRef Medline

Sasaki T, Takai Y (1998) The Rho small G protein family-Rho GDI system as a temporal and spatial determinant for cytoskeletal control. Biochem Biophys Res Commun 245:641-645. CrossRef Medline

Tang Y, Das U, Scott DA, Roy S (2012) The slow axonal transport of alphasynuclein-mechanistic commonalities amongst diverse cytosolic cargoes. Cytoskeleton 69:506-513. CrossRef Medline

Wujek JR, Lasek RJ (1983) Correlation of axonal regeneration and slow component B in two branches of a single axon. J Neurosci 3:243-251. Medline

Ylera B, Ertürk A, Hellal F, Nadrigny F, Hurtado A, Tahirovic S, Oudega M, Kirchhoff F, Bradke F (2009) Chronically CNS-injured adult sensory neurons gain regenerative competence upon a lesion of their peripheral axon. Curr Biol 19:930-936. CrossRef Medline

Zala D, Hinckelmann MV, Yu H, Lyra da Cunha MM, Liot G, Cordelières FP, Marco S, Saudou F (2013) Vesicular glycolysis provides on-board energy for fast axonal transport. Cell 152:479-491. CrossRef Medline 